\title{
FORTALECIMIENTO DE LA COMPETENCIA CIENTÍFICA IMPLEMENTANDO RUTINAS DE PENSAMIENTO EN EL COLEGIO COOPERATIVO COMFENALCO
}

\author{
STRENGTHENING SCIENTIFIC COMPETENCE BY \\ IMPLEMENTING THOUGHT ROUTINES AT THE \\ COLEGIO COOPERATIVO COMFENALCO
}

\author{
Estefanía Díaz-Ochoa ${ }^{1}$ \\ Natalia Rodríguez-López ${ }^{1}$
}

\section{RESUMEN}

Los análisis de las pruebas PISA y las pruebas saber en cuanto al pensamiento científico, indicaron que existe una carencia por parte de los estudiantes para interpretar, abordar situaciones problemas y construir alternativas de solución, lo cual genera inconsistencias en la educación colombiana. Por esta razón, el objetivo de esta investigación ha sido fortalecer la competencia científica en niños de 5- 6 años en una institución educativa de carácter privado, a través de una propuesta de intervención pedagógica basada en rutinas de pensamiento. A partir de lo anterior se establecieron los objetivos específicos: Identificar las habilidades científicas de los niños, a través de actividades diagnósticas; diseñar las intervenciones pedagógicas basadas en rutinas de pensamiento; e implementar y evaluar las actividades determinando la efectividad de las rutinas de pensamiento, en el fortalecimiento de las habilidades científicas.

El estudio es de tipo cualitativo con diseño metodológico de investigación acción, en la cual se describió y reflexionó sobre la práctica docente en la enseñanza de las ciencias desde la educación inicial. La propuesta concluyó con la importancia de incorporar rutinas de pensamiento en la educación inicial para fortalecer la competencia científica, dado que los niños de transi-

1 Universidad Autónoma de Bucaramanga - UNAB. Facultad de Ciencias Sociales Humanidades y Artes. Maestría en Educación. Bucaramanga, Colombia. 
ción evidenciaron avances en el desempeño académico durante y después de la implementación de la estrategia pedagógica, mostrándose más críticos e infiriendo coherentemente ante situaciones; además de tener mayor habilidad al clasificar objetos, indagar sobre la realidad, plantear hipótesis, planificar acciones para llegar a un fin y explicar resultados a través de la experimentación.

PALABRAS CLAVE: Rutinas de pensamiento, competencia científica, educación inicial.

\section{ABSTRACT}

The analysis of the PISA and Saber tests in terms of scientific thinking, indicated that there is a lack of students to interpret, address problems and construct alternative solutions, which generates inconsistencies in Colombian education. For this reason, the objective of this research was to strengthen scientific competence in children from 5 to 6 years of age in a private educational institution, through a proposal of pedagogical intervention based on thinking routines. Based on the above, the specific objectives were established; identifying children's scientific skills through diagnostic activities, designing pedagogical interventions based on thinking routines, and lastly, implementing and evaluating activities determining the effectiveness of thinking routines in the strengthening of scientific skills.

Guided by the qualitative paradigm and having as a methodological design the Action Research, which analyzed, described and reflected on the teaching practice in the teaching of science from the initial education, taking as a starting point, their knowledge, experiences and interactions with the social environment, and considering the role of the child as an active subject of rights in the educational context. The proposal concludes with the importance of incorporating routines of thought in the initial education to strengthen the scientific competence, since the transitional children evidenced advances in the academic performance during and after the implementation of the pedagogical strategy, showing more critical, coherently inferring faced with situations that challenged thought, as well as having greater ability to classify objects, to inquire about reality, to hypothesize, to plan actions to reach an end and to explain results through experimentation.

KEY WORDS: Routines of thought, scientific competence, initial education.

\section{INTRODUCCIÓN}

La presente investigación estableció como objetivo fortalecer la competencia científica en niños de 5 - 6 años en una institución educativa de carácter privado, a través de una propuesta de intervención pedagógica basada en rutinas de pensamiento, la cual respondió a la pregunta problema: ¿De qué manera la implementación de rutinas de pensamiento fortalece la competencia científica en niños de transición? El estudio se encuentra organizada en cinco capítulos; en el primero se dan a conocer los antecedentes, planteamiento del problema y justi- 
ficación, a partir de la reflexión sobre la importancia de la enseñanza de las ciencias y visibilización del pensamiento desde la educación inicial, por lo cual se establece la pregunta problema: ¿de qué manera las rutinas de pensamiento fortalecen la competencia científica en niños de 5 a 6 años? En este apartado también se establece el objetivo general y los específicos.

En el segundo capítulo se encuentra la fundamentación teórica que sustenta la investigación, desde la concepción de niño, primera infancia, enseñanza de las ciencias, competencia científica y rutinas de pensamiento. De igual forma, se presentan las investigaciones empíricas, las cuales dan un aporte al estudio. Posteriormente, en la tercera sección se expone la metodología de la investigación, basada en el paradigma cualitativo, con diseño metodológico investigación acción.

Seguidamente, en el cuarto apartado se presentan los resultados con su respectivo análisis, confiabilidad y validez de la información. En el quinto capítulo se retoma la pregunta problema para dar respuesta a esta y se establecen las conclusiones a partir de los objetivos específicos. Finalmente, se presenta la bibliografía.

\section{METODOLOGÍA}

Esta investigación se desarrolló bajo el paradigma cualitativo, en el cual se realizaron aproximaciones generales a las situaciones sociales y a fenómenos educativos, atendiendo al análisis, descripción y reflexión de la práctica docente en la enseñanza de las ciencias desde la educación inicial.

Considerando que se pretendió generar una propuesta de intervención para mejorar las prácticas pedagógicas en el desarrollo de las habilidades científicas de los niños de transición 1 del Colegio Cooperativo Comfenalco de la ciudad de Bucaramanga, se utilizó el diseño metodológico de Investigación Acción, desarrollado en las fases establecidas por Carr y Kemmis (1990): planificación, ejecución, observación y reflexión.

\section{ETAPA DE PLANIFICACIÓN}

Para efectuar la valoración inicial, se planearon 4 actividades diagnósticas con una duración de 30 minutos. Con cada actividad se evaluaron las habilidades científicas a fortalecer (observación, clasificación, inferencia, planificación, indagación, planteamiento de hipótesis, experimentación y explicación de sucesos). 
La propuesta para contribuir al fortalecimiento de la competencia científica en la educación inicial se desarrolló por medio de tres proyectos pedagógicos de aula, con un total de 20 actividades, las cuales giraron en torno al fortalecimiento de las habilidades científicas empleando las rutinas de pensamiento veo-piensome pregunto, yo pensaba... ahora pienso, zoom in, pensar-cuestionar-explorar.

\section{ETAPA DE EJECUCIÓN}

Un primer momento de ejecución, fue el desarrollo de las actividades que permitieron realizar las valoraciones de las condiciones de entrada de los 20 niños de transición 1; estas se llevaron a cabo durante dos semanas. En el primer semestre del año 2017 se ejecutaron los tres proyectos de aula, con una duración de tres meses. En total se llevaron a cabo 20 actividades de 30 a 50 minutos aproximadamente. Para su desarrollo se utilizaron tres días por semana, es decir, 12 intervenciones por mes.

\section{ETAPA DE OBSERVACIÓN}

En el transcurso de la investigación, la observación se convirtió en una técnica fundamental que permitió percibir y dar cuenta de las intervenciones de los docentes, de los intereses y necesidades de los niños. Igualmente, la observación se hizo presente en el desarrollo de todas las actividades, puesto que fue necesario percibir los comportamientos, respuestas y actitudes de los niños con respecto a las intervenciones diarias e interacción efectuada, teniendo en cuenta las categorías y subcategorías de análisis en la implementación de rutinas de pensamiento para el fortalecimiento de las habilidades científicas en niños de $5-6$ años.

\section{ETAPA DE REFLEXIÓN}

Se generó la reflexión a partir del análisis de los resultados en cada intervención pedagógica, para dar cuenta de la importancia de las rutinas de pensamiento en la enseñanza de las ciencias y en cualquier área; todo ello dio lugar al mejoramiento de la práctica pedagógica y la transformación en el contexto educativo.

\section{TÉCNICAS E INSTRUMENTOS}

En el transcurso del proceso se hizo presente la observación, la cual permitió percibir y describir el comportamiento de los participantes en un ambiente o escenario natural. Para ello se empleó la rejilla de observación, como instrumento que permitió hacer la valoración de las condiciones de entrada según el 
desarrollo de las actividades diagnósticas. El instrumento se construyó a partir de los diversos planteamientos y teorías que sustentaron el aprendizaje y desarrollo de los infantes en la competencia científica. A su vez, este instrumento sirvió para determinar el fortalecimiento de las habilidades científicas de los niños al utilizar las rutinas de pensamiento en cada proyecto de aula.

Teniendo en cuenta que de manera permanente se interactuó con los niños, percibiendo e interpretando sus intereses, habilidades, dificultades, ideas y procesos, se implementó la observación participante. Durante el ejercicio de observación, interacción y participación en el proceso investigativo, se utilizó el diario pedagógico para realizar los registros y análisis del comportamiento, preguntas, desempeño y comentarios denotados por los niños.

Después de ejecutar cada actividad en los proyectos de aula, se escogían aleatoriamente a 6 niños y se conformaba un grupo focal con el fin de conocer su percepción sobre la intervención y la implementación de la rutina de pensamiento.

Al culminar los proyectos de aula se realizó una entrevista a 5 padres de familia, con el fin de dar cuenta de la trascendencia de los temas abordados y del fortalecimiento de las habilidades científicas en los niños que hicieron parte del proceso.

Se empleó una entrevista estructurada, de la que se obtuvieron los siguientes resultados:

En esta se estipulan los logros e indicadores de las habilidades científicas a fortalecer, así como se muestra en la tabla 2.

El primer momento, comprendió el diseño, implementación y análisis de las intervenciones pedagógicas basadas en las rutinas de pensamiento, en el fortalecimiento de las habilidades científicas. Para el proceso de análisis se definieron las categorías de análisis, las cuales apuntaron a dos aspectos esenciales, el primero, al trasfondo pedagógico y didáctico de la implementación de la rutina de pensamiento, para lo cual se estableció la siguiente categoría y subcategorías: Planificación de la enseñanza empleando rutinas de pensamiento: pertinencia, estructura, tiempo, coherencia y pertinencia de los logros e indicadores.

Posteriormente, se orientó hacia las respuestas y resultados de los niños de acuerdo a la dimensión cognitiva, específicamente en las habilidades científicas. Esta categoría se denominó: evaluación en el fortalecimiento de las habilidades científicas empleando rutinas de pensamiento, la cual comprendió evaluación de la enseñanza y evaluación del aprendizaje como subcategorías. 
Tabla 2

REJILLA DE OBSERVACIÓN

\begin{tabular}{|c|c|c|c|c|}
\hline Dimensiones & Categoría & Subcategoría & Logros & Indicadores \\
\hline \multirow[t]{3}{*}{ D. Cognitiva } & \multirow[t]{3}{*}{$\begin{array}{l}\text { Habilidades } \\
\text { científicas }\end{array}$} & Observación & $\begin{array}{l}\text { Utiliza los } \\
\text { sentidos con } \\
\text { seguridad para } \\
\text { obtener } \\
\text { información de lo } \\
\text { que lo rodea en } \\
\text { su entorno. }\end{array}$ & $\begin{array}{l}\text { - Utiliza un solo rasgo perceptivo para tratar de } \\
\text { comprender de forma global lo que son las } \\
\text { cosas. } \\
\text { - Selecciona la información relevante de entre } \\
\text { lo irrelevante en el contexto de un determinado } \\
\text { problema. } \\
\text { - Dirige su atención de manera consciente y la } \\
\text { mantiene dirigida hacia determinados objetos y } \\
\text { situaciones orientadas por la acción del adulto. } \\
\text { - Presta atención a los detalles y no solo a las } \\
\text { características que saltan a la vista, en especial } \\
\text { si son objetos de su interés. } \\
\text { - Observa las semejanzas y diferencias y y } \\
\text { compara los objetos según sus características. } \\
\text { - Explora los elementos y objetos de su entorno. } \\
\text { - Utiliza la observación para explicar y } \\
\text { comprender las causas de un fenómeno. } \\
\text { - Observa los objetos, fenómenos o hechos y yo } \\
\text { establece la relación causa-efecto. }\end{array}$ \\
\hline & & Clasificación & $\begin{array}{l}\text { Identifica } \\
\text { semejanzas y } \\
\text { diferencias para } \\
\text { organizar objetos } \\
\text { en grupos y } \\
\text { subgrupos } \\
\text { atendiendo a sus } \\
\text { características. }\end{array}$ & $\begin{array}{l}\text { - Reconoce las características comunes entre } \\
\text { los objetos, para agruparlos formando una } \\
\text { colección. } \\
\text { - Agrupa objetos siguiendo más de dos criterios. } \\
\text { - Selecciona los objetos haciendo comparacio- } \\
\text { nes entre sus propiedades. } \\
\text { - Explica la organización del material } \\
\text { atendiendo a las propiedades de la colección. } \\
\text { - Reúne elementos con cualidades comunes } \\
\text { formando grupos y subgrupos de una categoria } \\
\text { inicial. }\end{array}$ \\
\hline & & Indagación & $\begin{array}{l}\text { Formula } \\
\text { preguntas sobre } \\
\text { los objetos, } \\
\text { organismos y } \\
\text { fenómenos, y a } \\
\text { su vez, explora } \\
\text { posibles } \\
\text { respuestas para } \\
\text { interpretar su } \\
\text { entorno y } \\
\text { construir el } \\
\text { conocimiento. }\end{array}$ & $\begin{array}{l}\text { - Conduce sus preguntas a la reflexión y } \\
\text { construcción del conocimiento. } \\
\text { - Formula preguntas de acuerdo con la } \\
\text { situación planteada. } \\
\text { - Expresa sus inquietudes, curiosidad e } \\
\text { intereses por medio de la pregunta. } \\
\text { - Escucha las preguntas planteadas por sus } \\
\text { pares. } \\
\text { - Observa detalladamente y pregunta por las } \\
\text { causas o razones de las situaciones. } \\
\text { - Hago conjeturas para responder mis pre- } \\
\text { guntas. } \\
\text { - Expone preguntas que orienta a la búsqueda, } \\
\text { exploración y experimentación. } \\
\text { - Relaciona una situación con otra a través de } \\
\text { preguntas. } \\
\text { - Busca respuesta a las preguntas a través de } \\
\text { sus experiencias e ideas previas. } \\
\text { - Da respuesta a sus preguntas consultando, } \\
\text { manipulando y explorando su entorno. } \\
\text { - Da respuesta a las preguntas planteadas por } \\
\text { sus pares. } \\
\text { - Busca respuesta a sus preguntas cuestio- } \\
\text { nando a los otros. } \\
\text { - Selecciona la información adecuada para dar } \\
\text { respuesta a sus preguntas. }\end{array}$ \\
\hline
\end{tabular}


Tabla 2 (Cont.)

\begin{tabular}{|c|c|c|c|c|}
\hline Dimensiones & Categoría & Subcategoría & Logros & Indicadores \\
\hline \multirow[t]{5}{*}{ D. Cognitiva } & \multirow[t]{5}{*}{$\begin{array}{l}\text { Habilidades } \\
\text { científicas }\end{array}$} & Inferencia & $\begin{array}{l}\text { Organiza sus } \\
\text { ideas para } \\
\text { generar } \\
\text { soluciones y dar } \\
\text { explicaciones en } \\
\text { distintos } \\
\text { contextos. }\end{array}$ & $\begin{array}{l}\text { - Comprende situaciones del entorno. } \\
\text { - Realiza inferencias con respuestas precisas a la } \\
\text { situación. } \\
\text { - Establece relaciones entre los objetos de } \\
\text { acuerdo con sus características. } \\
\text { - Busca la relación causa-efecto entre los } \\
\text { fenómenos o situaciones. } \\
\text { - Representa mentalmente las cosas de su } \\
\text { realidad. } \\
\text { - Encuentra analogías entre objetos y sucesos. } \\
\text { - Se enfoca en los detalles de la imagen llegandoa } \\
\text { conclusiones lógicas. } \\
\text { - Extrae conclusiones sobre las situaciones, } \\
\text { objetos y fenómenos sin ser directamente } \\
\text { observables. } \\
\text { - Presenta la capacidad de buscar y analizar la } \\
\text { información. } \\
\text { - Explica la situación de acuerdo con la } \\
\text { problemática presentada. }\end{array}$ \\
\hline & & $\begin{array}{l}\text { Planificación } \\
\text {. }\end{array}$ & $\begin{array}{l}\text { Establece } \\
\text { acciones para } \\
\text { conseguir un fin } \\
\text { determinado de } \\
\text { acuerdo con sus } \\
\text { intereses y las } \\
\text { situaciones } \\
\text { presentadas. }\end{array}$ & $\begin{array}{l}\text { - Actúa de manera ordenada en el desarrollo de } \\
\text { las actividades. } \\
\text { - Anticipa los hechos de acuerdo a las situaciones } \\
\text { que se le presentan. } \\
\text { - Reconoce que existe una secuencia de acciones } \\
\text { para cumplir metas. } \\
\text { - Explica los pasos necesarios para cumplir un } \\
\text { objetivo. } \\
\text { - Registro mis observaciones y el plan de acción } \\
\text { en forma organizada y rigurosa (sin alteraciones), } \\
\text { utilizando dibujos, palabras y números. }\end{array}$ \\
\hline & & $\begin{array}{l}\text { Planteamiento } \\
\text { de hipótesis }\end{array}$ & $\begin{array}{l}\text { Formula } \\
\text { hipótesis para } \\
\text { explorar, } \\
\text { analizar, explicar } \\
\text { fenómenos y dar } \\
\text { soluciones a las } \\
\text { diferentes } \\
\text { situaciones } \\
\text { problemas que } \\
\text { se presenten en } \\
\text { su entorno. }\end{array}$ & $\begin{array}{l}\text { - Establece suposiciones de acuerdo a } \\
\text { problemáticas planteadas. } \\
\text { - Construye relaciones sobre hechos y fenómenos } \\
\text { de la realidad. } \\
\text { - Establece significados propios para dar } \\
\text { explicación de lo que sucede. } \\
\text { - Relaciona eventos para predecir resultados. }\end{array}$ \\
\hline & & Experimentación & $\begin{array}{l}\text { Demuestra } \\
\text { interés por } \\
\text { explorar los } \\
\text { espacios y } \\
\text { objetos para } \\
\text { conocer y } \\
\text { comprender todo } \\
\text { aquello que lo } \\
\text { rodea. }\end{array}$ & $\begin{array}{l}\text { - Descubre nuevas acciones para comprobar } \\
\text { hipótesis. } \\
\text { - Comprende características de objetos. } \\
\text { - Describe situaciones problemáticas y trabaja en } \\
\text { pro de las soluciones. } \\
\text { - Establece resultados a partir de la experimenta- } \\
\text { ción con objetos y fenómenos del medio. } \\
\text { - Identifica la validez o invalidez de las hipótesis. }\end{array}$ \\
\hline & & $\begin{array}{l}\text { Explicación } \\
\text { de resultados }\end{array}$ & $\begin{array}{l}\text { Comunica sus } \\
\text { ideas y } \\
\text { conocimientos } \\
\text { para dar } \\
\text { explicaciones } \\
\text { lógicas a los } \\
\text { fenómenos y } \\
\text { resultados } \\
\text { obtenidos. }\end{array}$ & $\begin{array}{l}\text { - Expresa con claridad las soluciones para llegar a } \\
\text { un resultado. } \\
\text { - Establece conclusiones en la solución de } \\
\text { situaciones problemáticas. } \\
\text { - Socializa con sus pares y docentes los } \\
\text { resultados obtenidos durante el proceso. } \\
\text { - Expone sus puntos de vista, teniendo en cuenta } \\
\text { sus pre-saberes para llegar al resultado obtenido. }\end{array}$ \\
\hline
\end{tabular}

Fuente: propia. 


\section{RESULTADOS}

Para dar cumplimiento al objetivo de la investigación fue necesario valorar las condiciones de entrada de los niños de transición 1 del colegio Cooperativo Comfenalco, por medio de las 4 actividades diagnósticas que giraron en torno a las habilidades a fortalecer en la investigación. A continuación, se presentan los resultados por habilidad:

\section{OBSERVACIÓN}

En términos generales los niños hicieron uso de la observación para describir los objetos e imágenes que se les presentaron, atendiendo a detalles que saltaban a la vista, además, por medio de la observación comenzaron a hacer relaciones de causa-efecto sobre la situación planteada.

\section{CLASIFICACIÓN}

El grupo en general demostró características de la etapa de colecciones figurales comprendida a los 4 años, en la que reúne elementos formando una figura, aunque también puede hacerlo siguiendo un atributo. Estas especificaciones no corresponden a sus edades ( $5-6$ años) puesto que a los 5 años deben clasificar siguiendo dos o tres criterios y a los 6 años se clasifica estableciendo semejanzas, diferencias e inclusión, dando explicación de su distribución, lo que se denomina "Clasificación lógica u operatoria" (Labinowicz, 2000).

\section{INDAGACIÓN}

Se percibió que, a partir de un ejemplo o explicación de formular las preguntas, los niños tuvieron la capacidad de hacer indagaciones relacionando situaciones y expresando sus inquietudes.

\section{INFERENCIA}

En la actividad "La expresión del arcoíris" se evidenció que los niños establecieron diferentes respuestas referentes a la aparición del arcoíris, demostrando limitaciones del pensamiento descritas por Piaget en la etapa pre operacional, específicamente el artificialismo y finalismo, considerando que atribuyeron la creación del arcoíris a un ser supremo y con un fin determinado.

Solo 2 niños del grupo atendieron a sus conocimientos previos frente a la situación y se acercaron a la explicación correcta al expresar lo siguiente: "El arcoíris sale cuando llueve y sale el sol"; "el arcoíris sale porque llueve y hace sol al tiempo". 
Por otra parte, en las respuestas de los niños frente a las inquietudes del grupo (“ipor qué los sapos salen cuando llueve?", “ipor qué las nubes están negras?", “icómo nace el arcoíris?”) tuvieron la capacidad de extraer conclusiones según la situación, establecer relación de causa y efecto, explicar un suceso y representar mentalmente los hechos de su realidad.

\section{PLANIFICACIÓN}

Se evidenciaron falencias en la habilidad de planificación puesto que inicialmente los niños no concibieron las acciones y el procedimiento a seguir para conseguir el tesoro.

\section{PLANTEAMIENTO DE HIPÓTESIS Y EXPERIMENTACIÓN}

En la actividad "la pista correcta" los niños plantearon diferentes hipótesis al determinar la pista en que alcanzaría mayor distancia el carro. Sus hipótesis hacían alusión a la forma y textura de los materiales presentados, por lo cual, establecieron significados según rasgos perceptivos, ello corresponde a su desarrollo cognitivo. Con las acciones repetitivas de los niños, demostraron interés por explorar los espacios y objetos para comprobar sus hipótesis.

\section{EXPLICACIÓN DE RESULTADOS}

Al comprobar las hipótesis de los niños por medio de la experimentación, ellos explicaron los resultados teniendo en cuenta sus pre-saberes, estableciendo relaciones y analogías.

Después de valorar las condiciones de entrada de los niños por medio de las 4 actividades diagnósticas, se diseñó la propuesta de intervención pedagógica basada en rutinas de pensamiento que permitieran el fortalecimiento de las habilidades científicas en niños de 5 a 6 años. Finalizadas las intervenciones pedagógicas correspondientes a los tres proyectos de aula, se diseñó la rejilla en la que se evidencia la pertinencia y selección de las actividades implementadas, denotando a su vez observaciones generales de efectividad y mejoramiento.

Después de determinar la pertinencia se realizó el análisis de las actividades atendiendo a las categorías y subcategorías establecidas. En cada una de estas se evaluó la efectividad de las rutinas de pensamiento en el fortalecimiento de las habilidades científicas, para lo cual se tuvo en cuenta la estructura, acciones efectuadas, cumplimiento de los objetivos, práctica docente y percepciones de los 
Tabla 3

\section{PERTINENCIA Y SELECCIÓN DE LAS ACTIVIDADES}

\begin{tabular}{|c|c|c|c|c|}
\hline Proyecto de aula & Actividad & $\mathbf{S i}$ & No & Observaciones generales \\
\hline \multirow[t]{8}{*}{$\begin{array}{l}\text { Proyecto 1: } \\
\text { Cuido mi } \\
\text { ambiente }\end{array}$} & $\begin{array}{l}\text { Act. } \mathrm{N}^{01} \mathrm{La} \\
\text { imagen } \\
\text { escondida. }\end{array}$ & & $\mathrm{X}$ & $\begin{array}{l}\text { En la rutina de pensamiento "zoom in" las partes de la } \\
\text { imagen eran muy evidentes, impidiendo la observación de } \\
\text { detalles, similitudes y el establecimiento de diferencias y } \\
\text { semejanzas en las representaciones. De igual forma, faltó } \\
\text { que las docentes promovieran la curiosidad, creatividad e } \\
\text { inferencia a través de preguntas relacionadas a la imagen } \\
\text { en su conjunto. }\end{array}$ \\
\hline & $\begin{array}{c}\text { Act. } \mathrm{N}^{\circ} 2 \\
\text { Todos a limpiar }\end{array}$ & $\mathrm{x}$ & & $\begin{array}{l}\text { Se promovió la participación y expresión oral de los niños, } \\
\text { generando un ambiente agradable e interés por el } \\
\text { tratamiento de los residuos sólidos en el proceso de } \\
\text { reciclaje. }\end{array}$ \\
\hline & $\begin{array}{l}\text { Act. } \mathrm{N}^{\circ} 3 \mathrm{La} \\
\text { basura en su } \\
\text { lugar }\end{array}$ & $\mathrm{x}$ & & $\begin{array}{l}\text { En el desarrollo de la actividad se evidenciaron los } \\
\text { indicadores correspondientes a la habilidad de observación } \\
\text { y clasificación, así como fue planificado. }\end{array}$ \\
\hline & $\begin{array}{l}\text { Act. } N^{\circ} 4 \\
\text { Ayudemos a } \\
\text { Nano }\end{array}$ & & $x$ & $\begin{array}{l}\text { En la actividad se cumplió con lo esperado (Identificar los } \\
\text { puntos ecológicos y clasificar los residuos sólidos según } \\
\text { corresponda) no obstante, el saber adquirido fue puesto en } \\
\text { práctica en la actividad "guardianes ambientes", siendo } \\
\text { esta más completa. }\end{array}$ \\
\hline & $\begin{array}{l}\text { Act. } N^{\circ} 5 \\
\text { Guardianes } \\
\text { ambientales. }\end{array}$ & $x$ & & $\begin{array}{l}\text { El juego de roles y el diseño del emblema alusivo a "los } \\
\text { guardines ambientales" promovió la participación y el } \\
\text { interés por enseñarle a los otros la distribución correcta de } \\
\text { los residuos sólidos. Por tanto, se dio cumplimiento a los } \\
\text { logros e indicadores respectivos. }\end{array}$ \\
\hline & $\begin{array}{c}\text { Act } N^{\circ} 6 \mathrm{El} \\
\text { paso a paso. }\end{array}$ & $\mathrm{X}$ & & $\begin{array}{l}\text { En la actividad los niños evocaron y representaron el } \\
\text { procedimiento para reciclar los residuos sólidos, } \\
\text { demostrando habilidad en el proceso de planificación }\end{array}$ \\
\hline & $\begin{array}{l}\text { Act N }{ }^{0} 7 \text { Ayuda } \\
\text { al ambiente. }\end{array}$ & & $X$ & \multirow{2}{*}{$\begin{array}{l}\text { Los niños estuvieron interesados en el diseño del cohete } \\
\text { con material reciclable, permitiendo el cumplimiento de los } \\
\text { objetivos de la actividad, sin embargo, la trascendencia en } \\
\text { las habilidades científicas fue mínima. }\end{array}$} \\
\hline & $\begin{array}{l}\text { Act } \mathrm{N}^{\circ} 8 \mathrm{Tu} \\
\text { idea realidad. }\end{array}$ & & $x$ & \\
\hline \multirow[t]{6}{*}{$\begin{array}{l}\text { Proyecto 2: } \\
\text { Germinación de } \\
\text { la semilla }\end{array}$} & $\begin{array}{l}\text { Act. } N^{\circ} 1 \\
\text { Semilla } \\
\text { escondida. }\end{array}$ & & $\mathrm{X}$ & $\begin{array}{l}\text { En la actividad los niños se apropiaron de las funciones de } \\
\text { las partes de la planta, sin embargo, el impacto generado } \\
\text { fue muy poco, teniendo en cuenta que tenían un } \\
\text { conocimiento previo del tema. }\end{array}$ \\
\hline & $\begin{array}{l}\text { Act. } N^{\circ} 2 \text { ¿Qué } \\
\text { hay dentro de } \\
\text { la semilla? }\end{array}$ & $\mathrm{x}$ & & $\begin{array}{l}\text { El espacio, los recursos y materiales empleados llamaron la } \\
\text { atención de los niños, promoviendo la participación y el } \\
\text { interés por explorar y representar gráficamente el } \\
\text { contenido de la semilla. }\end{array}$ \\
\hline & $\begin{array}{l}\text { Act. } \mathrm{N}^{\circ} 3 \mathrm{El} \\
\text { sembrado de } \\
\text { las semillas. }\end{array}$ & $\mathrm{X}$ & & $\begin{array}{l}\text { La actividad fue globalizadora pues permitió el } \\
\text { fortalecimiento de la habilidad de observación, } \\
\text { planteamiento de hipótesis e indagación. A su vez, se } \\
\text { evidenciaron los indicadores correspondientes a los } \\
\text { procesos. }\end{array}$ \\
\hline & $\begin{array}{l}\text { Act. } \mathrm{N}^{\circ} 4 \\
\text { Alimento para } \\
\text { las plantas. }\end{array}$ & $\mathrm{x}$ & & $\begin{array}{l}\text { Gracias a la actividad hubo un equilibrio entre los saberes } \\
\text { previos y el conocimiento adquirido por los niños, logrando } \\
\text { la apropiación y comprensión de los factores que } \\
\text { intervienen en el crecimiento de las plantas. }\end{array}$ \\
\hline & $\begin{array}{l}\text { Act. N }{ }^{\circ} 5 \text { Los } \\
\text { cambios en la } \\
\text { germinación. }\end{array}$ & $\mathrm{x}$ & & $\begin{array}{l}\text { A partir de los registros en la bitácora, los niños fueron } \\
\text { conscientes del proceso de germinación de la semilla de } \\
\text { frijol y lenteja, estableciendo comparaciones y relaciones } \\
\text { de causa-efecto. }\end{array}$ \\
\hline & $\begin{array}{l}\text { Act } N^{2} 6 \text { La flor } \\
\text { nuestra. }\end{array}$ & & $\mathrm{X}$ & $\begin{array}{l}\text { Fue la materialización del proyecto, por lo cual no se } \\
\text { planificó el fortalecimiento de habilidades científicas. }\end{array}$ \\
\hline
\end{tabular}


Tabla 2 (Cont.)

\begin{tabular}{|c|c|c|c|c|}
\hline Proyecto de aula & Actividad & Si & No & Observaciones generales \\
\hline \multirow[t]{6}{*}{$\begin{array}{l}\text { Proyecto 3: } \\
\text { El mundo de } \\
\text { los alimentos }\end{array}$} & $\begin{array}{l}\text { Act. } N^{0} 1 \text { La } \\
\text { oxidación de } \\
\text { las frutas. }\end{array}$ & & $\mathrm{X}$ & $\begin{array}{l}\text { Se extendió por varios días la actividad interfiriendo en su } \\
\text { continuidad y en la atención y disposición de los niños }\end{array}$ \\
\hline & $\begin{array}{l}\text { Act. } N^{\circ} 2 \\
\text { El mundo de } \\
\text { las frutas. }\end{array}$ & $x$ & & $\begin{array}{l}\text { Se evidenció interés y destreza en la clasificación de las } \\
\text { frutas atendiendo a más de dos criterios y a su vez en la } \\
\text { formación de grupos y subgrupos. }\end{array}$ \\
\hline & $\begin{array}{l}\text { Act. } \mathrm{N}^{\circ} 3 \\
\text { Uvas } \\
\text { bailarinas. }\end{array}$ & $x$ & & $\begin{array}{l}\text { Fue una actividad que llamó y mantuvo la atención de los } \\
\text { niños en los momentos de intervención. Además, se dio } \\
\text { cumplimiento a los indicadores correspondientes al } \\
\text { proceso de observación, planteamiento de hipótesis e } \\
\text { indagación. }\end{array}$ \\
\hline & $\begin{array}{l}\text { Act. } N^{\circ} 4 \\
\text { Lo más } \\
\text { pesado. }\end{array}$ & $x$ & & $\begin{array}{l}\text { Los materiales empleados despertaron el interés de los } \\
\text { niños por formular hipótesis, manipular y hacer la } \\
\text { experimentación respectiva, logrando así la comprensión } \\
\text { de densidad. }\end{array}$ \\
\hline & $\begin{array}{l}\text { Act. N }{ }^{\circ} 5 \\
\text { Alimentación } \\
\text { saludable. }\end{array}$ & $X$ & & $\begin{array}{l}\text { La actividad permitió la reflexión de los niños sobre los } \\
\text { alimentos que consumen diariamente, promoviendo la } \\
\text { alimentación saludable en la escuela y en el hogar. }\end{array}$ \\
\hline & $\begin{array}{l}\text { Act } N^{\circ} 6 \\
\text { Recetas } \\
\text { saludables. }\end{array}$ & & $\mathrm{X}$ & $\begin{array}{l}\text { Con la rutina de pensamiento "yo pensaba....ahora pienso" } \\
\text { hubo dificultad para establecer el pensamiento anterior y } \\
\text { actual, basado en el procedimiento de las recetas } \\
\text { realizadas y la alimentación saludable. }\end{array}$ \\
\hline
\end{tabular}

niños según los grupos focales realizados al finalizar cada actividad. Posterior al análisis, reflexión y mejoramiento de las actividades se dio a conocer la propuesta en una cartilla con el fin de contribuir a la práctica pedagógica en la enseñanza de las ciencias y al fortalecimiento de las habilidades científicas en la educación inicial.

\section{TRIANGULACIÓN DE RESULTADOS}

De acuerdo con las actividades diagnósticas y cada una de las intervenciones pedagógicas basada en rutinas de pensamiento, se puede determinar que los niños avanzaron en las habilidades de clasificación, planificación, explicación de resultados, inferencias e indagación, considerando que inicialmente presentaron falencias en el desarrollo de las actividades. Una de las causas de estas dificultades fueron las limitaciones del pensamiento que caracterizan la etapa preoperacional correspondiente a sus edades. 
Sin embargo, por medio de las actividades y a partir de su desarrollo cognitivo los niños lograron hacer una representación de su realidad considerando diferentes perspectivas que le permitían justificar, explicar y describir las situaciones presentadas. Este comportamiento también demuestra la etapa preoperacional, puesto que se caracteriza por "la descomposición del pensamiento en función de imágenes, símbolos y conceptos, las acciones se hacen internas a medida que puede representar cada vez mejor un objeto o evento por medio de su imagen mental y de una palabra" (Labinowicz 2000, p.67).

Cabe mencionar que en las actividades con rutinas de pensamiento se evidenció la habilidad de los niños para plantear hipótesis, experimentar y hacer uso de la observación. Estos procesos y los anteriores, se fueron fortaleciendo y potenciando en el transcurso de los proyectos de aula, demostrando características de la competencia científica descritas por Caño y Luna (2011), es decir los niños desarrollaron la capacidad para emplear el conocimiento, identificar preguntas, hacer conjeturas, obtener información, concluir y analizar su entorno, con el fin de comprender los fenómenos naturales y los cambios que en ocasiones dependen de la actividad humana.

Teniendo en cuenta que en cada actividad se pretendía fortalecer las habilidades científicas, se hace una descripción de los logros alcanzados según el planteamiento teórico:

A través de la observación los niños utilizaron en su totalidad los sentidos para identificar, reconocer, comparar y contrastar elementos, permitiendo la comprensión y explicación de los fenómenos. González y García (2014) afirmaron que las acciones anteriores definen la observación.

La observación facilitó la formulación de preguntas sobre los objetos, organismos y fenómenos. Sin embargo, su planteamiento no fue inmediato sino un proceso continuo de mejoramiento, en el que se superó la dificultad presentada ante la estructura y formulación respectiva.

En otra instancia, se reconoce que las preguntas formuladas facilitaron la experimentación y generó interés por explorar los espacios y objetos para buscar respuestas, interpretar todo aquello que lo rodea y construir el conocimiento.

La interpretación de los niños condujo al planteamiento de hipótesis, Puche et al. (2001) expresó que este proceso cognitivo se evidencia de forma temprana, ya que la "constante búsqueda de respuestas y su curiosidad lo llevan permanentemente a formular hipótesis ante los diferentes fenómenos" (p.20). 
Así como lo expresan los autores, los niños establecieron hipótesis en las actividades respectivas, realizando la experimentación para determinar su validez y concluir sobre el caso, teniendo en cuenta los niños desde los quince meses, tienen la capacidad de crear, generar y variar sus acciones para conseguir un fin o responder ante una nueva situación (Restrepo, 2007).

No obstante, el tipo de experimentación presentado y que corresponde a sus edades es la procedural (Ordoñez, 2003), asumiendo que aún no tienen la capacidad mental para explicar y argumentar de manera científica los diferentes fenómenos. Por esta razón fue válido que los niños se basaran en sus conocimientos previos e intereses para explicar coherentemente las situaciones percibidas.

Finalmente, en la habilidad de planificación lo niños inicialmente no se detuvieron a pensar en el plan ni en el procedimiento de las situaciones planteadas, lo cual corresponde a su desarrollo cognitivo, así como lo plantea Osorio (2009): los niños "solo planean el primer paso y a partir del resultado de este, piensan qué hacer después; esta puede entenderse como planeación a corto plazo, la planeación a mediano y largo plazo la construyen con la maduración cognitiva" (p. 37). No obstante, a partir de las actividades se logró que percibieran los pasos para conseguir un fin determinado, representando y nombrando las acciones para alcanzar el objetivo en situaciones específicas.

Para abarcar otra fuente de información dentro de la triangulación, se tuvo en cuenta la descripción realizada por los padres de familia en las entrevistas, con el fin de determinar la trascendencia de las intervenciones pedagógicas y los avances en los niños durante la investigación, específicamente en las habilidades científicas.

En primera instancia, los padres manifestaron que los niños anteriormente cuestionaban sobre aspectos de su entorno, pero durante las intervenciones estuvieron más interesados en formular preguntas constantemente, puesto que planteaban interrogantes basados en la temática de los tres proyectos "Cuido mi ambiente", "Germinó la semilla" y "El mundo de los alimentos", remitiéndose a interrogantes como: "idebo llevar la misma lonchera saludable?", "imami qué estás haciendo con la basura?”, ipor qué la gente bota basura en la calle?, "imami por qué de un frijol que se comen salen pelos? Y "iPor qué el agua cuando está con el algodón comienza a salirle la florecita?".

En cuanto a la forma de responder a los cuestionamientos que se plantean, los padres expresaron que los niños buscan la manera de conocer la realidad, ya sea por medio de intervención familiar, análisis de lo observado, con ayuda tecno- 
lógica o relacionando sus pre saberes. Con esto se evidencia que existe mayor motivación e interés por encontrar respuestas, indagar y hacer uso de otros medios para validar lo que han aprendido.

También expresaron que los niños se han mostrado muy sensibles, curiosos, se asombran con facilidad y analizan situaciones poco comunes. De igual forma, los padres evidenciaron avances durante el proceso, expresando que los niños ponen en práctica lo visto en el colegio respecto al reciclaje y consumo de alimentos saludables, manifestando apropiación de los temas. Finalmente, los padres afirmaron que los niños logran concluir sobre situaciones que han observado y analizado previamente, demostrando interés por indagar, conocer e investigar más.

\section{CONCLUSIONES}

Atendiendo al primer objetivo correspondiente a la identificación de las condiciones de entrada de los niños de transición 1 en la competencia científica, se concluye que, a pesar de su corta edad y su desarrollo cognitivo, los niños presentan habilidades científicas que les permiten explorar su entorno, observar, dar explicaciones y resolver situaciones, teniendo como referencia sus conocimientos y experiencias previas. Su curiosidad y el interés por manipular, percibir y escudriñar lo desconocido y los seres que lo rodean, lo llevan a indagar, plantear hipótesis y a establecer respuestas que lo conducen a la construcción de diversos saberes acordes con su edad. Es así como se demuestra lo expresado por Restrepo (2007) y Puche, et al. (2001), al afirmar que los niños poseen habilidades científicas en diferentes niveles de desarrollo, que facilitan la resolución de problemas.

Sin embargo, el procedimiento de las habilidades científicas, como la planificación, inferencia, explicación de resultados y clasificación, son más complejos, puesto que requiere una madurez respectiva y superación de las limitaciones del pensamiento según la etapa de desarrollo en que se encuentre, es este caso la etapa pre operacional establecida por Piaget (2001).

A partir del segundo objetivo referente al diseño de la propuesta de intervención pedagógica basada en rutinas de pensamiento, se concluye que el trabajo por proyectos de aula favorece la relación entre los saberes y el medio, reconociendo el desarrollo e integración de diferentes procesos y competencias, que conduce al niño a interpretar y dar soluciones a las situaciones y problemáticas del contexto. Es así como se es coherente con lo expresado por Rincón (2012), 
quien afirmó que el trabajo por proyectos es una propuesta que integra los aprendizajes y busca que el conocimiento responda a las necesidades de la realidad. Por lo tanto, el trabajo por proyectos no solo responde a las necesidades del contexto, sino que también crea nuevos ambientes de aprendizaje, promueve el desarrollo integral de los niños, genera la participación y despierta el interés por reconocer su entorno (MEN, 2010).

Asimismo, se concluye que para el diseño de la propuesta de intervención pedagógica es importante identificar las características de los niños según su etapa de desarrollo con el fin de plantear de manera coherente los logros y los indicadores. De igual forma, se requiere claridad en el procedimiento de la rutina para que respondan al fortalecimiento de las habilidades científicas, de acuerdo a las condiciones de entrada y al proceso de los niños.

En cuanto a la implementación de rutinas de pensamiento, se estipula que estas juegan un papel importante en el ámbito educativo, puesto que además de fortalecer el pensamiento científico se contribuye al desarrollo de otras habilidades como la oralidad, atención, creatividad, escucha y respeto en los espacios de participación. Por consiguiente, incluir rutinas de pensamiento en las actividades posibilita un trabajo transversal en las dimensiones del desarrollo. No obstante, la efectividad de la rutina de pensamiento dependerá del conocimiento previo de su procedimiento y la orientación adecuada del docente.

Atendiendo al último objetivo del proyecto sobre la evaluación de las actividades pedagógicas, determinando la efectividad de las rutinas de pensamiento en el fortalecimiento de las habilidades científicas, se concluye que, mediante el análisis basado en las categorías y subcategorías establecidas en el proyecto, se logró hacer una valoración de cada intervención, según la coherencia y cumplimiento de los logros e indicadores, la estructura ordenada, tiempo y rol del docente. Lo anterior constituye una parte importante dentro del proyecto, puesto que, a través de ello, se realizó la selección de las actividades más pertinentes para fortalecer la competencia científica en niños de 5 a 6 años. 


\section{Entrevista $\mathrm{N}^{\circ} 1$}

\section{Preguntas a padres de familia del grado transición}

\section{1. ¿Ha notado que su hijo (a) formula preguntas constantemente? ¿Cuáles?}

$\mathrm{R} /$ "Si, ella vive muy pendiente de lo que sucede, sobre todo de las noticias que habla. Hace preguntas como, por ejemplo: ¿aquí también nos vamos a inundar?, ¿mamá y si tiembla qué vamos a hacer, si no estoy contigo, si estoy en el colegio?

Con la comida ahora está muy pendiente, me pregunta ¿debo llevar la misma lonchera saludable?, ¿por qué las papas es comida chatarra?, mami es que la profe me dice que las Minichips son comida chatarra y que yo debo llevar comida saludable y traer todos los días una fruta". También me preguntó ¿mami será que la semilla crece más rápido porque sucede eso?, ¿si nos inundamos es porque tiramos basuras y tapamos los ríos?

Ahora ella hace más preguntas, sobre todo de ¿cómo nacen o se hacen los niños? Porque quiere tener un hermanito"

2. ¿De qué manera su hijo (a) encuentra respuestas a las preguntas que formula?

R/ "Ella espera a que yo le conteste, pero me da más opciones"

\section{3. ¿Cómo es el actuar de su hijo (a) frente a una situación poco común?}

R/ "Ella empieza a preguntarme, pero es muy sensible frente a los fenómenos o catástrofes naturales.

Frente a otras situaciones, por ejemplo, con la Pony Malta, cuando se la mando yo le saco un poquito el gas, entonces dice si mami porque se me riega y la boto toda. Ella en una ocasión que la Pony Malta se le regó por el gas, trataba era de cerrarla, actúa a que no siga pasando.

También observa bien lo que está pasando, si no entiende me pregunta a mí, o ella misma inventa cosas para decir lo que sucede, esto se acerca, pero también es muy imaginaria, trata de dar respuestas, pero con cositas que uno dice eso no es, pero ella trata de explicar algo".

\section{4. ¿Qué avances en la dimensión cognitiva has notado en tu hijo(a)?}

$\mathbf{R}$ / $\mathrm{Si}$, por ejemplo, en lo que le digo de los alimentos, porque está más pendiente de qué comida le sirve y qué no. Yo la llevo a natación entonces, está muy pendiente de hacer deporte.

Está más pendiente del reciclaje, sin que yo le diga lleva las bolsas plásticas donde es, también con las inundaciones, terremotos, está muy pendiente de eso. Lo del agua, a todos, nos dice que no debemos botar el agua. Pone en práctica todo lo que la profe le enseña, nos lo aplica a nosotros". 


\section{REFERENCIAS BIBLIOGRÁFICAS}

Caño, A. y Luna, G. (2011). Competencia científica para el mundo de mañana: Marco y análisis del ítem. Bilbao: Instituto Vasco de Evaluación e Investigación Educativa.

Carr, W. y Kemmis, S. (1990). Teoría crítica de la enseñanza la investigación-acción en la formación del profesorado. Barcelona: Martínez Roca.

Carrero, G. (2006). La Educación científica en el nivel preescolar de la educación inicial desde la percepción del mediador pedagógico. (Tesis doctoral) Universidad Industrial de Santander. Bucaramanga.

Chávez, L. y Romero, R. (2011). Desarrollo de habilidades de pensamiento inferencial y comprensión de la lectura en niños de tres a seis años. Panorama, 11, 103-125.

Coll, C., Palacios, J., y Marchesi, A. (1998). Desarrollo Psicológico y educación. Psicología evolutiva. Madrid: Alianza.

Condemarín, M., Chadwick, M. y Milicic, N. (1986). Madurez Escolar. Chile: Andres Bellos.

Decastro, M. (2012). Las rutinas de pensamiento: una estrategia para visibilizar mi aprendizaje. (Tesis de Maestría). Universidad de la Sabana, Bogotá.

Flavell, J. (1998). La psicología evolutiva de Jean Piaget. Buenos Aires: Paidós.

García, M. y Peña, P. (2002). Los encuentros científicos en preescolar. Educere, 6 (19), 308-315.

Gil, D., Carrascosa, J., Furió, C., y Martínez, J. (1991). La enseñanza de las ciencias en la educación secundaria: planteamientos didácticos generales y ejemplos de aplicación en las ciencias físicoquímicas Col. Cuadernos de Educación (5). Barcelona.

Gonzalez, A. y Fuentes, M. (1995). Psicologia del desarrollo: Málaga: Aljibe.

González, D. y García, Y. (2014). Desarrollo de habilidades científicas en la formación inicial de profesores de ciencias y matemática.

Henao, M. y Ramírez, O. (2015). Estrategia para favorecer el desarrollo de la Competencia Científica Identificar. Universidad del valle. Obtenido de

Instituto Colombiano para el Fomento de la Educación Superior -ICFES. (2013). Colombia en pisa 2012, Informe nacional de resultados. Bogotá. Colombia.

Instituto Colombiano para el Fomento de la Educación Superior -ICFES. (s.f.). Módulo de Pensamiento científico Matemáticas y Estadística Saber Pro 2016-2. Bogotá, Colombia.

Labinowicz, E. (2000). Introducción a Piaget. México: Calipso.

Mejía, MR, y Manjarrés, ME (2007). La pregunta como punto de partida y estrategia pedagógica. Colombia: Colciencias.

Ministerio de Educación Nacional. (2004a). Cómo formar científicos sociales y naturales. Al tablero 30. Bogotá: Editorial Magisterio.

Ministerio de Educación Nacional. (2004b). Estándares Básicos de Competencias en Ciencias Naturales y Ciencias Sociales. Formar en ciencias: iEl desafío! Bogotá: Editorial Magisterio. 
Ministerio de Educación Nacional. (2010). Orientaciones Pedagógicas para el Grado de Transición (borrador). EDESCO Ltda. Dirección de Calidad para la Educación Preescolar, Básica y Media por medio de su subdirección de referentes y evaluación de la calidad educativa. Bogotá: Editorial Magisterio.

Oppenheimer, A. (2010) iBasta de Historias! La obsesión latinoamericana con el pasado y las 12 claves del futuro. Colombia: Géminis.

Ordóñez, O. (2003). Hipótesis, experimento e inferencias en el niño. Una propuesta de análisis. En R. Puche y otros, El niño: Científico, lector y escritor, matemático (2ae ed.). Santiago de Cali: Universidad del Valle/Artes Gráficas del Valle.

Ortega, R. (1999). Crecery aprender. Aprendizaje. España: Visor.

Ortiz, G. y Cervantes, M. (2015). La formación científica en los primeros años de escolaridad. Panorama, 9 (17), 10-23.

Osorio, A. (2009). Habilidades científicas de los niños y niñas participantes en el programa de pequeños científicos de Manizales. [Tesis de Maestría]. Universidad de Manizales.

Papalia,D. y Wendkos, S. (1997). Desarrollo humano. Sexta edición. México: McGraw Hill.

Pasek, E. y Matos, Y. (2007). Habilidades cognitivas básicas de investigación presentes en el desarrollo de los proyectos de aula. Educere, 11 (37), 349-356.

Perkins, D., Tishman, S. y Jay, E. (1998). Un aula para pensar: Aprender y enseñar en una cultura del pensamiento. Buenos Aires. Aique.

Piaget, J. (1972). El nacimiento de la inteligencia en el niño. Madrid: Aguilar.

Piaget, J. (2001). La representación del mundo en el niño. Novena Edición. Madrid: Morata.

Pinzón, Y. y Póveda, M. (2016). Potenciando la voz de los niños a través de rutinas de pensamiento. (Tesis de Maestría). Universidad de la Sabana, Bogotá.

Puche, R., Colinvaux, D. y Dibar, C. (2001). El niño que piensa. Un modelo de transformación de maestros. Universidad del Valle. Colombia: Artes gráficas del Valle Editores

Quintanilla, M. (2012). Las Competencias de Pensamiento Científico desde 'las voces' del aula. Vol. 1 Universidad Católica de Chile.

Quiroga, M., Arredondo, E., Cafena, D. y Merino, C. (2014). Desarrollo de competencias científicas en las primeras edades: El Explora Conicyt de Chile. Educ.Educ. 17 (2), 237-253.

Restrepo, F. (2007). Habilidades investigativas en niños y niñas de 5 a 7 años de Instituciones oficiales y privadas de la ciudad de Manizales. Doctorado en ciencias sociales. Niñez y juventud. Pontificia Universidad Javeriana. Manizales, Colombia.

Rincón, G. (2012). Los proyectos de aula y la enseñanza y el aprendizaje del lenguaje escrito. Tejer la RED/3.

Ritchhart, R., y Perkins, D. (2008). Making Thinking Visible (2008). Educational Leadership, 65 (5), 57-61.

Roncancio, N. (2012). Revisión sistemática acerca de las competencias investigativas en primera infancia. Horizontes Pedagógicos, 14 (1). 119-134/ISSN: 0123-8264. 
Rubinstein, SL (1958). El Pensamiento y los caminos de su investigación. U.R.S.S.

Segura, D. (2011). El pensamiento científico y la formación temprana: una aproximación a las prácticas escolares en los primeros años vistas desde la ciencia y la tecnología. Nodos y Nudo, 3 (31), 4-15.

Trujillo, E. (2007). Propuesta metodológica para la alfabetización científica de niños en edad preescolar. Revista Anales de la Universidad Metropolitana, (7) 1, 73-93.

UNESCO. (1999). Declaración sobre la ciencia y el uso del saber científico. Adoptada por la conferencia mundial sobre las ciencias.

Vygostky, L. (1979). El desarrollo de los procesos psicológicos superiores. Barcelona: Crítica. 